\title{
Results of single-probe microwave ablation of metastatic liver cancer ${ }^{\text {th }}$
}

\author{
R. Hompes ${ }^{\mathrm{c}}$, S. Fieuws ${ }^{\mathrm{a}, \mathrm{d}}$, R. Aerts ${ }^{\mathrm{c}}$, M. Thijs ${ }^{\mathrm{b}}$, F. Penninckx ${ }^{\mathrm{c}}$, B. Topal ${ }^{\mathrm{c}, *}$

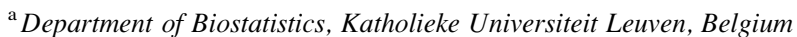 \\ ${ }^{\mathrm{b}}$ Department of Radiology, University Hospital Leuven, Herestraat 49, 3000 Leuven, Belgium \\ ${ }^{\mathrm{c}}$ Department of Abdominal Surgery, University Hospital Leuven, Herestraat 49, 3000 Leuven, Belgium \\ ${ }^{\mathrm{d}}$ Department of Biostatistics, Universiteit Hasselt, Belgium
}

Accepted 10 May 2010

Available online 3 June 2010

\begin{abstract}
Aims: Microwave ablation (MWA) is the most recent development in the field of local ablative therapies. The aim of this study was to evaluate the variability and reproducibility of single-probe MWA vs. radiofrequency ablation (RFA) of liver metastases smaller than $3 \mathrm{~cm}$ in patients without underlying liver disease.

Methods: Sixteen liver metastases were treated using MWA, and matched for size and localisation with 13 metastases treated by RFA. Tumour diameters and postoperative ablation diameters were recorded (D1 transverse; D2 antero-posterior; D3 cranio-caudal; mm) on computed tomography scans.

Results: Median D1, D2, and D3 ablation diameters after MWA vs. RFA were $18.5(12-64)$ vs. 34 (16-41) mm ( $p=0.003)$, 26 (14-60) vs. $35(28-40) \mathrm{mm}(p=0.046)$, and $20(10-73)$ vs. $32(20-45) \mathrm{mm}(p=0.025)$, respectively. As compared to RFA, the variability between the lesions after MWA was significantly higher for D2 $(p<0.0001)$ and D3 $(p=0.002)$ but not for D1 $(p=0.15)$. The ablation diameters were less uniform after MWA than after RFA $(p<0.001)$.

Conclusion: Ablation diameters after single-probe MWA of metastatic liver tumours are highly variable and suboptimal. Improvements are needed before MWA can be implemented routinely.
\end{abstract}

(C) 2010 Elsevier Ltd. All rights reserved.

\section{Introduction}

Surgical resection is the gold standard for treatment of primary or metastatic liver cancer. However, most patients are not candidates for hepatic resection due to anatomic limitations, multifocal nature of the disease, insufficient functional liver reserve, extra-hepatic metastases or comorbidities. Consequently, a number of interstitial or local ablative therapies (LAT) have been developed and gained popularity for the treatment of unresectable liver tumours. Radiofrequency ablation (RFA) has become an established treatment modality due to its efficacy, reproducibility, low

\footnotetext{
ClinicalTrials.gov identifier: NCT00922181.

* Corresponding author. Department of Abdominal Surgery, University Hospital Leuven, Herestraat 49, 3000 Leuven, Belgium. Tel.: +32 16 344265; fax: +32 16344832 .

E-mail address: baki.topal@med.kuleuven.be (B. Topal).
}

complication rates and availability. ${ }^{1,2}$ However, high local recurrence rates particularly for lesions larger than $3 \mathrm{~cm}$ in diameter are a point of concern. ${ }^{2-4}$ Further criticism has focused on the potential for incomplete tumour ablation near large vessels due to the heat-sink effect, slow rate of heating and the small zone of active heating requiring multiple overlapping probe placements to achieve larger ablation zones. $^{5-7}$ Therefore, technological advancements resulted in the development of new local ablative modalities such as the microwave.

Microwave ablation (MWA) has several theoretical advantages over RFA, which may make it more attractive than RFA to treat hepatic tumours. The benefits of MWA are an improved convection profile, higher constant intratumoural temperatures, faster ablation times and the ability to use multiple probes to treat multiple lesions simultaneously. Moreover, MWA does not require the placement of grounding pads that can result in skin burns. The size 
and shape of the MWA zone may be more consistent and less dependent on the heat-sink effect from vascular structures in proximity of the lesion. ${ }^{8,9}$ Thus MWA may overcome some known limitations of RFA and may result in higher efficacy.

Any LAT should provide long-term complete tumour destruction with predictable and reproducible results. There are few studies available, all on animal models, reporting conflicting results on the size and geometry of the coagulation zone obtained with MWA vs. RFA. ${ }^{10,11}$ The aim of the current study was to evaluate the variability and reproducibility of ablation diameters obtained after single-probe MWA vs. RFA in patients with liver metastases smaller than $3 \mathrm{~cm}$ without underlying liver disease.

\section{Patients and methods}

\section{Patients and tumours}

Liver metastases measuring less than $3 \mathrm{~cm}$ in diameter were considered for the current study. From August 2008 until November 2008, single-probe MWA was used to treat 16 liver metastases in 6 patients without underlying liver disease (4 female and 2 male; median age 64 years, range 47-82). Four patients underwent MWA for 10 liver metastases from colorectal cancer. Patients with colorectal liver metastases were considered for MWA but not for surgical resection since they had a high clinical risk score (CRS 3 or more), ${ }^{12}$ showed none to minimal response to systemic chemotherapy, and suffered from severe systemic disease (ASA 3 or more).

One patient was treated for a solitary liver metastasis from lung cancer, and another patient for 5 metastases from cancer of the ampulla Vateri. Segmental localisation of liver metastases was as follows: 1 in $\mathrm{Sg} 4$ (segment), 6 in $\mathrm{Sg} 5,2$ in $\mathrm{Sg} 6,3$ in $\mathrm{Sg} 7$, and 4 in $\mathrm{Sg} 8$.

Within our liver database, metastases treated with MWA were matched for size and localisation with 12 liver metastases from colorectal cancer and 1 from cervical cancer, which were treated with RFA in 13 patients (6 female and 7 male; median age 58 (35-70) years). Segmental localisation of liver metastases in the RFA group was as follows: 1 in $\mathrm{Sg} 4,4$ in $\mathrm{Sg} 5,3$ in $\mathrm{Sg} 7$, and 5 in $\mathrm{Sg} 8$.

\section{Surgical procedure}

MWA was performed via laparoscopy in 5 patients to treat 15 liver metastases and percutaneously in 1 patient with a solitary lesion. In 1 patient with colorectal liver metastases laparoscopic MWA was combined with segmental hepatectomy. A single cooled percutaneous/laparoscopic microwave antenna of $22 \mathrm{~cm}$ length and $3.7 \mathrm{~cm}$ active radiating tip (VT2237) was used with a $915 \mathrm{MHz}$ Valleylab MW ablation generator (VTSYS3; Covidien, Europe). Microwave energy was applied for $10 \mathrm{~min}$ at $40 \mathrm{~W}$, according to the manufacturer's guidelines.
RFA was performed for 15 min per tumour using a monopolar $200 \mathrm{~W}$ RF generator and a single cool-tip laparoscopic electrode of $25 \mathrm{~cm}$ length and $3 \mathrm{~cm}$ activating tip (Covidien, Radionics Europe NV) as described earlier. ${ }^{13}$ Radiofrequency ablation was performed by laparoscopy in 7 patients, by laparotomy in 4 (with simultaneous colorectal surgery), and percutaneously in 2 patients.

At all times, intra-operative ultrasound was used for positioning the MWA antenna or the RFA needle electrode. Continuous ultrasound monitoring was performed to track the progress of the ablation. The extent of the ablated coagulation could be roughly approximated on the basis of the appearance of the transient hyperechoic zone.

\section{Assessment of outcome measures}

Pre-operative tumour diameters and postoperative ablation diameters, assessed by dual-phase contrast enhanced helical computed tomography (CT) liver scan, were recorded (D1 transverse; D2 antero-posterior; D3 cranio-cau$\mathrm{dal} ; \mathrm{mm})$. Microwave ablation diameters were compared with those of matched RF ablated tumours. Postoperative measurements were performed within one week and at 3 months after surgery. Irregular peripheral contrast enhancement and a multilobular shape at the ablation margin were considered as a criterion for residual tumour on 3-phase CT-scan of the liver. Local recurrence was defined as cancer recurrence at the site of ablated liver metastasis.

\section{Statistical analysis}

MWA and RFA were compared with respect to the increase in lesion diameters measured before and after treatment, the variability between the lesions after ablation, and the variability within the lesion diameters.

Mann-Whitney U tests were used to compare measured diameters. A linear model was used to compare the changes between pre-operative diameters and postoperative ablation zones between both groups. A logarithmic transformation was needed to meet the statistical assumptions of the model. To assess differences in variability of the post-treatment measurements, likelihood-ratio tests were used (using maximum likelihood estimation) comparing linear models fitted on the post-treatment measurements. Included lesions were considered as independent units. All analyses were performed using the statistical software SAS (version 9.2). A $p$-value $\leq 0.05$ was considered statistically significant.

\section{Results}

\section{Tumour and ablation diameters}

The median transverse (D1), antero-posterior (D2), and cranio-caudal (D3) tumour diameters before MWA vs. RFA were 12 (range $6-18)$ vs. $12(7-24) \mathrm{mm}, 12(6-24)$ vs. 12 (7-17) $\mathrm{mm}$, and $10.5(6-20)$ vs. $11(8-20) \mathrm{mm}$, respectively 
$(p>0.792)$. The median $\mathrm{D} 1, \mathrm{D} 2$, and $\mathrm{D} 3$ ablation diameters after MWA vs. RFA were $18.5(12-64)$ vs. $34(16-41) \mathrm{mm}$ $(p=0.003), 26(14-60)$ vs. $35(28-40) \mathrm{mm}(p=0.046)$, and $20(10-73)$ vs. $32(20-45) \mathrm{mm}(p=0.025)$, respectively (Fig. 1). The increase in all 3 diameters was more pronounced after RFA as compared to that after MWA, and most apparent for the transverse diameter (Table 1). For the 3 dimensions taken together, the diameter increased 2.6 times (95\% confidence interval CI: 2.3-3.1) after RFA compared to 2.1 times (95\% CI: $1.8-2.5)$ after MWA ( $p=0.058)$.

As compared to RFA, the variability between the lesion diameters after MWA was significantly higher for D2 $(p<0.0001)$ and D3 $(p=0.002)$ but not for D1 $(p=0.15)$. The degree of uniformity, i.e. the variability between the three diameters within a lesion, was significantly higher after MWA than after RFA $(p<0.001)$.

\section{Clinical outcome and radiological assessment}

No peri-operative mortality was observed. After MWA, one patient developed haemobilia that resolved conservatively. According to the CT-scan performed within 1 week after MWA, the overall tumour destruction was complete. In all these patients the zone of ablation extended into the liver capsule at the site of MWA-probe insertion. This phenomenon, known as the 'comet effect', is illustrated in Fig. 2. The median follow-up time after MWA was 6.3 (4.9-7.8) months, and was complete. One biopsy proven local recurrence was observed after MWA in a patient treated for a solitary colorectal liver metastasis. No recurrences were observed in the RFA group.

\section{Discussion}

\section{Local recurrence after ablation of liver tumours}

The majority of patients with hepatic malignancies are not candidates for surgical resection. For selected patients with unresectable disease LAT has become an accepted
Table 1

The increase in diameters after microwave vs. radiofrequency ablation of liver tumours

\begin{tabular}{llrl}
\hline Dimension $(\mathrm{mm})$ & RFA & \multicolumn{1}{l}{ MWA } & $p$-value \\
\hline Transverse & $22.0(4-27)$ & $9.0(2-47)$ & 0.006 \\
Antero-posterior & $24.0(18-30)$ & $13.7(5-42)$ & 0.058 \\
Cranio-caudal & $18.0(8-31)$ & $11.0(0-53)$ & 0.034 \\
\hline
\end{tabular}

The increase in diameters is presented as median (range) in millimetres; MWA: microwave ablation; RFA: radiofrequency ablation.

therapeutic modality to provide local tumour control, with the best opportunity for long-term survival. ${ }^{14,15}$ Currently, radiofrequency energy is the most widely used modality for the thermal ablation of liver tumours. The main criticism on RFA has focused on recurrence rates varying from $2 \%$ to $60 \% .{ }^{16-18}$ Local recurrence is related to the lack of complete coagulative necrosis and the persistence of residual tumour cells in or around the ablation zone. ${ }^{19,20}$ Independent risk factors for local recurrence after LAT are tumour size larger than $3 \mathrm{~cm}$, percutaneous approach, and the lack of a curative intention to treat. ${ }^{21,22}$

The limits in size of the coagulation zone that can be created by LAT, the irregular borders of both the tumour and the LAT zone, and the presence of satellite lesions around tumours, all contribute to incomplete tumour ablation. ${ }^{23-26}$ Blood vessels in the proximity of liver tumours can cause a heat-sink effect of local blood flow, resulting in local recurrence. ${ }^{5,21}$ The importance of coagulating a peri-tumoural margin of $1 \mathrm{~cm}$ has been highlighted in histological and clinical studies. ${ }^{21,22}$ Not only the size of the ablation zone matters but also the geometry of the lesion. ${ }^{27}$ Viable tumour cells can be present at the margin when the coagulation zone is asymmetric or eccentric. $^{25,28}$ Unfortunately, size and shape of radiofrequencyinduced ablations of liver tissue cannot be objectively evaluated intra-operatively. ${ }^{29}$ Indeed, usually a LAT device is chosen, according to the size of the tumoural lesion, which is supposed to yield an ablation zone of a determined size greater than the malignant lesion. In this regard, it is important that variability of size and shape of necrosis induced by
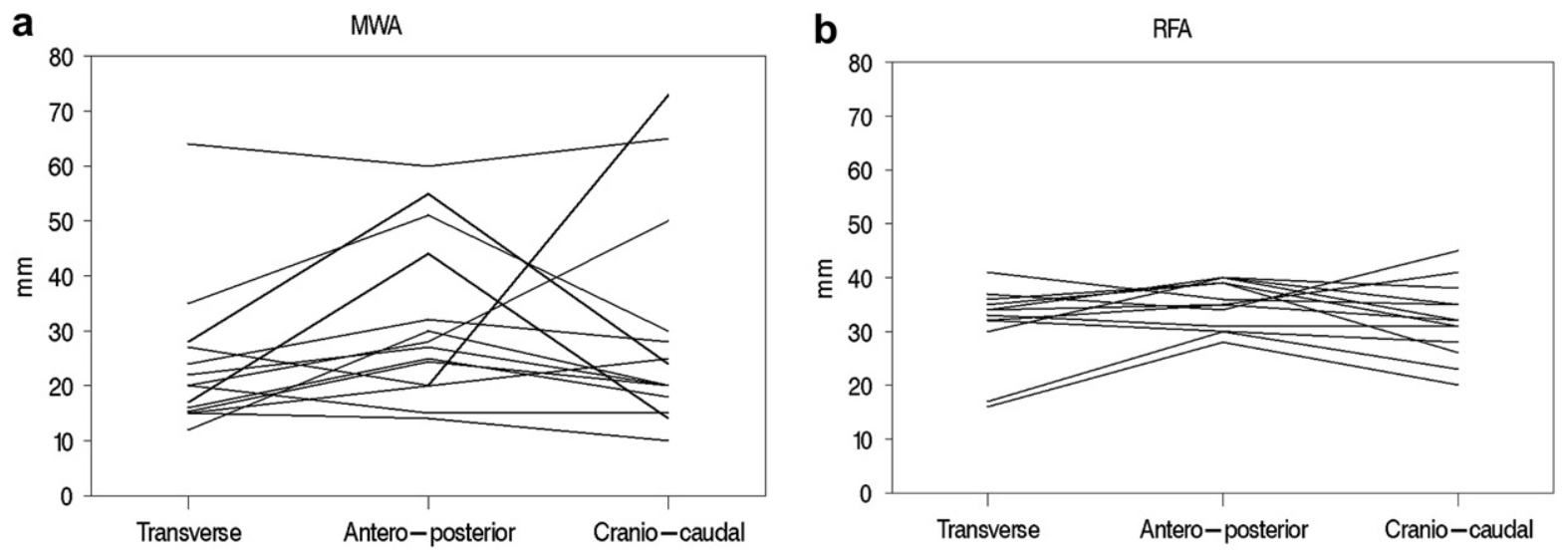

Figure 1. Diameters after microwave (a) and radiofrequency ablation (b) of liver tumours. 

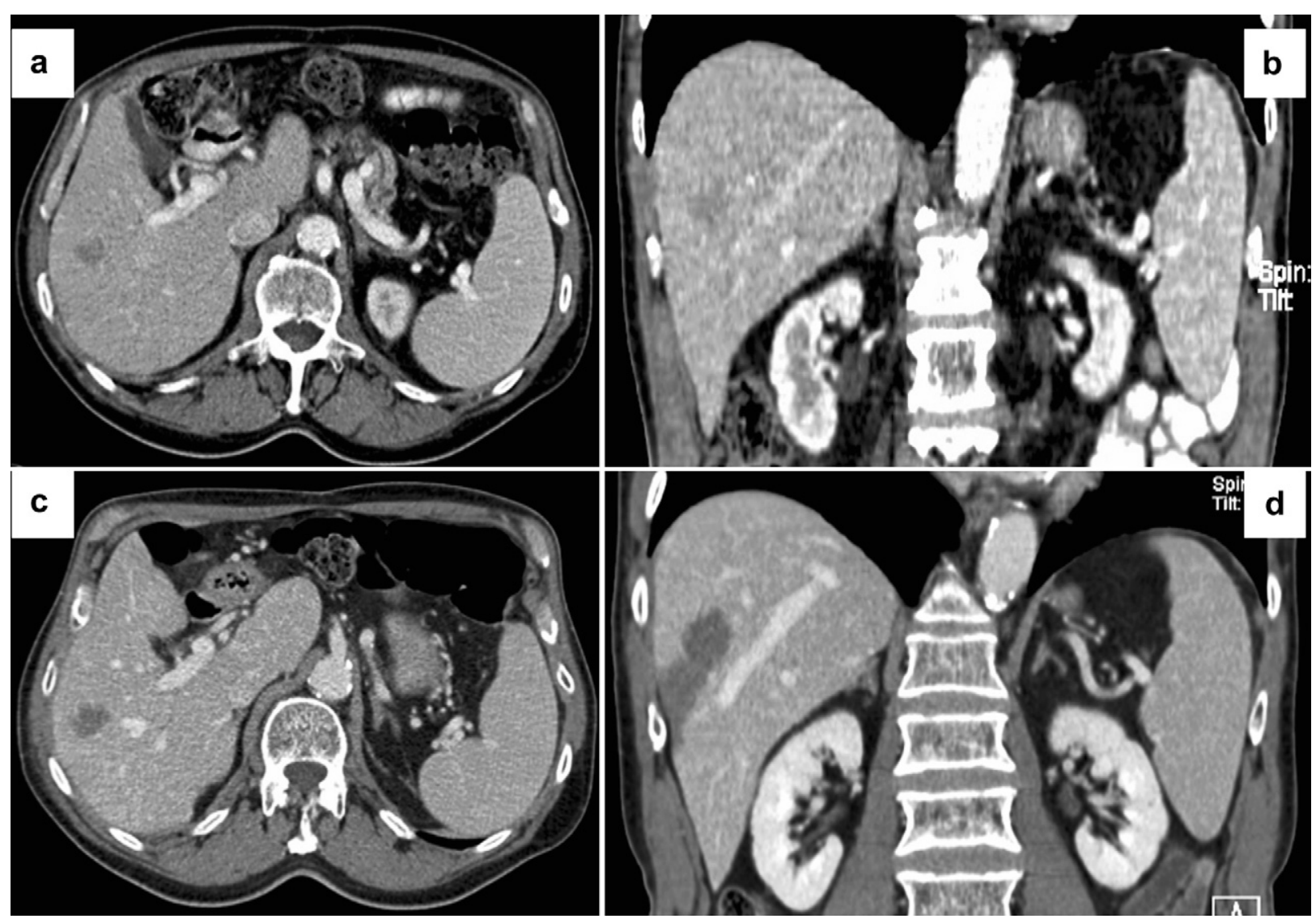

Figure 2. The 'comet effect' after microwave ablation (MWA) of liver tumours. Computed tomography scan showing a solitary liver metastasis from colorectal cancer in hepatic segment 5 , in axial $(a, c)$ and coronal $(b, d)$ view before $(a+b)$ and after $(c+d)$ MWA.

any LAT technique is low in order to achieve reproducible coagulation zones.

\section{Microwave ablation of liver tumours}

The application of microwave energy is the most recent development in the field of tumour ablation. The technique can be used percutaneously, via laparoscopy or via open surgical access. Microwave ablation offers many of the benefits of RFA but has some theoretical advantages that may result in improved performance. Compared with RFA, microwave ablation has a broader field of power density, resulting in a larger zone of active heating. ${ }^{30}$ In some reports the MWA zone can be up to $6 \mathrm{~cm}$ surrounding the MW antenna, allowing for a large volume of cell destruction within the targeted zone. ${ }^{8,30-32}$ Unlike RF energy, MW energy does not appear to be limited by charring and tissue desiccation, ${ }^{30}$ while the size and shape of the MWA zone may be more consistent and less dependent on the heat-sink effect from vascular structures in proximity. ${ }^{10,33,34}$ The knowledge on size and geometry of the coagulation zone is another determinant of local recurrence after LAT. ${ }^{35}$ Reports on the size of lesions obtained with MWA vs. RFA are conflicting and little is known about the geometry of the lesions. ${ }^{10,11}$

\section{Ablation diameters and variability}

In the present study, the comparison between MWA and RFA lesions was based on the analysis of the increase in lesion diameters measured before and after the ablation, the variability between the lesions after ablation, and the variability within a lesion diameter. The increase in all 3 diameters was more pronounced after RFA as compared to that after MWA. The lowest increase was measured for the transverse diameter after MWA, resulting in a final transverse diameter of $18.5 \mathrm{~mm}$. A final coagulation diameter of the MWA zone of less than $2 \mathrm{~cm}$ will inevitably lead to local cancer recurrence, as was observed in one patient in the current study, unless the tumour to be treated is smaller than $1 \mathrm{~cm}$. The variability between the lesions as well as the non-uniformity within one lesion diameter were higher after MWA as compared to RFA, making MWA less reproducible. Thus, the largest ablation diameters were obtained after RFA, with minimal variability in diameters between different lesions and high uniformity of the diameters within each lesion. The 'comet effect' of MWA was demonstrated by radiologic findings and confirmed by statistical comparison of the cranio-caudal diameters obtained after MWA vs. RFA. This dimension was the largest diameter obtained after MWA, and strongly 
dependent on the distance between the tumour and the site of probe insertion through the liver capsule.

In the current study, a single-probe MWA or RFA procedure was performed in all patients. Several authors reported that larger ablations can be obtained with multiple or clustered MW antennas. ${ }^{31,33,34,36}$ Simultaneous multiple-probe MWA could result in more uniform coagulation zones and better performance near blood vessels, and might lead to a more adequate treatment and decreased recurrence rates after tumour ablation. ${ }^{8,31}$ However, while multipleprobe MWA is able to achieve higher ablation volumes, it can also cause an increased 'comet effect' and unwanted collateral damage to neighbouring organs or structures. ${ }^{8}$

\section{Ablation energy source}

Limitations of the current study are the short follow-up time and its non-randomized nature. Nevertheless, before a randomized controlled trial is conducted to assess the oncologic outcome of MWA vs. RFA, their local effectiveness in terms of complete tumour destruction and complication rate should be evaluated. In the current study, the small transverse diameter obtained with MWA clearly raises concerns about the completeness of destruction even of small liver tumours. This finding was supported by the occurrence of a local recurrence within 6 months after MWA. One patient developed haemobilia after MWA of a solitary liver metastasis, which might be related to the unpredictable collateral damage caused by the 'comet effect' rather than to surgical expertise. ${ }^{13,22,37}$ One could also debate the use of different energy sources. Since both ablative techniques were used for a finite time at a specific setting, the amount of energy supplied by RFA was about eight times as high as the energy supplied by MWA. However, we believe that this reflects the difference in the physics of microwaves and radiofrequency. Thus a direct relation between energy supply and tissue damage is not clear. It is possible that increased power or longer times of MW application may create greater or more uniform lesions, but this was not addressed by our study. Second, we only looked at the performance of the new $915 \mathrm{MHz}$ Valleylab MW ablation system. An extensive experience with MWA comes from Asia where most microwave antennae operate at $2450 \mathrm{MHz}$. However, the $915 \mathrm{MHz}$ system that we used is more tuned to the dielectric properties of human tumour tissue. A comparative study of MWA zones between antennae operating at $2450 \mathrm{MHz}$ and $915 \mathrm{MHz}$, showed larger ablation zones with $915 \mathrm{MHz}$ microwaves. ${ }^{38}$ Another aspect of LAT modalities is their cost, provided they can ascertain similar short-term clinical and long-term oncologic outcomes.

\section{Conclusion}

Ablation diameters after single-probe MWA of liver metastases are highly variable and suboptimal. The small transverse diameter after MWA raises concerns about the completeness of destruction in tumours larger than $10 \mathrm{~mm}$, while the 'comet effect' may cause unwanted collateral damage to neighbouring organs or structures. Improvements are needed before MWA can be implemented routinely in clinical practice.

\section{Conflict of interest statement}

All authors have no conflict of interest, and disclose any potential or actual personal, political or financial interest in the material, information or techniques described in the paper.

\section{References}

1. Bilchik AJ, Wood TF, Allegra D, et al. Cryosurgical ablation and radiofrequency ablation for unresectable hepatic malignant neoplasms: a proposed algorithm. Arch Surg 2000;135:657-62.

2. Sutherland LM, Williams JA, Padbury RT, Gotley DC, Stokes B, Maddern GJ. Radiofrequency ablation of liver tumors: a systematic review. Arch Surg 2006;141:181-90.

3. Ng KK, Poon RT, Lo CM, Yuen J, Tso WK, Fan ST. Analysis of recurrence pattern and its influence on survival outcome after radiofrequency ablation of hepatocellular carcinoma. $J$ Gastrointest Surg 2008;12:183-91.

4. van Duijnhoven FH, Jansen MC, Junggeburt JM, et al. Factors influencing the local failure rate of radiofrequency ablation of colorectal liver metastases. Ann Surg Oncol 2006;13:651-8.

5. Kim SK, Rhim H, Kim YS, et al. Radiofrequency thermal ablation of hepatic tumors: pitfalls and challenges. Abdom Imaging 2005;30:72733.

6. Chin SB, Lee FT, Kennedy GD, et al. Effect of vascular occlusion on radiofrequency ablation of the liver: results in a porcine model. Am J Roentgenol 2001;176:789-95.

7. Goldberg SN, Gazelle GS, Solbiati L, Rittman WJ, Mueller PR. Radiofrequency tissue ablation: increased lesion diameter with a perfusion electrode. Acad Radiol 1996;3:636-44.

8. Wright AS, Lee FT, Mahvi DM. Hepatic microwave ablation with multiple antennae results in synergistically larger zones of coagulation necrosis. Ann Surg Oncol 2003;10:275-83.

9. Awad MM, Devgan L, Kamel IR, Torbensen M, Choti MA. Microwave ablation in a hepatic porcine model: correlation of CT and histopathologic findings. HPB 2007;9:357-62.

10. Wright AS, Sampson LA, Warner TF, Mahvi DM, Lee FT. Radiofrequency versus microwave ablation in a hepatic porcine model. Radiology 2005;236:132-9.

11. Shibata T, Niinobu T, Ogata N. Comparison of the effects of in-vivo thermal ablation of pig liver by microwave and radiofrequency coagulation. J Hepatobiliary Pancreat Surg 2000;7:592-8.

12. Fong Y, Fortner J, Sun RL, Brennan MF, Blumgart LH. Clinical score for predicting recurrence after hepatic resection for metastatic colorectal cancer. Analysis of 101 consecutive cases. Ann Surg 1999;230: $309-21$.

13. Topal B, Aerts R, Penninckx F. Laparoscopic radiofrequency ablation of unresectable liver malignancies: feasibility and clinical outcome. Surg Laparosc Endosc Percutan Tech 2003;13:11-5.

14. Mulier S, Ruers T, Jamart J, Michel L, Marchal G. Radiofrequency ablation versus resection for resectable colorectal liver metastases: time for a randomized trial? Dig Surg 2008;25:445-60.

15. Lau WY, Lai EC. The current role of radiofrequency ablation in the management of hepatocellular carcinoma: a systematic review. Ann Surg 2009;249:20-5. 
16. Curley SA, Izzo F, Delrio P, et al. Radiofrequency ablation of unresectable primary and metastatic hepatic malignancies: results in 123 patients. Ann Surg 1999;230:1-8.

17. Pawlik TM, Izzo F, Cohen DS, Morris JS, Curley SA. Combined resection and radiofrequency ablation for advanced hepatic malignancies: results in 172 patients. Ann Surg Oncol 2003;10:1059-69.

18. Kuvshinoff BW, Ota DM. Radiofrequency ablation of liver tumors: influence of technique and tumor size. Surgery 2002;132:605-11.

19. Curley SA. Radiofrequency ablation of malignant liver tumors. Oncologist 2001;6:14-23.

20. Siperstein A, Garland A, Engle K, et al. Local recurrence after laparoscopic radiofrequency thermal ablation of hepatic tumors. Ann Surg Oncol 2000;7:106-13.

21. Mulier S, Ni Y, Jamart J, Ruers T, Marchal G, Michel L. Local recurrence after hepatic radiofrequency coagulation: multivariate meta-analysis and review of contributing factors. Ann Surg 2005;242:158-71.

22. Topal B, Aerts JL, Roskams T, et al. Cancer cell dissemination during curative surgery for colorectal liver metastases. Eur J Surg Oncol 2005;31:506-11.

23. Adam R, Hagopian EJ, Linhares M, et al. A comparison of percutaneous cryosurgery and percutaneous radiofrequency for unresectable hepatic malignancies. Arch Surg 2002;137:1332-9.

24. Livraghi T, Goldberg SN, Lazzaroni S, et al. Hepatocellular carcinoma: radio-frequency ablation of medium and large lesions. Radiology 2000;214:761-8.

25. Shirabe K, Takenaka K, Gion T, et al. Analysis of prognostic risk factors in hepatic resection for metastatic colorectal carcinoma with special reference to the surgical margin. Br J Surg 1997;84:1077-80.

26. Maeda T, Takenaka K, Adachi E, et al. Small hepatocellular carcinoma of single nodular type: a specific reference to its surrounding cancerous area undetected radiologically and macroscopically. J Surg Oncol 1995;60:75-9.

27. Mulier S, Ni Y, Miao Y, et al. Size and geometry of hepatic radiofrequency lesions. Eur J Surg Oncol 2003;29:867-78.
28. Pulvirenti A, Garbagnati F, Regalia E, et al. Experience with radiofrequency ablation of small hepatocellular carcinomas before liver transplantation. Transplant Proc 2001;33:1516-7.

29. Raman SS, Lu DSK, Vodopich DJ, Sayre J, Lassman C. Creation of radiofrequency lesions in a porcine model: correlation with sonography, CT, and histopathology. Am J Roentgenol 2000;175: 1253-8.

30. Skinner MG, Iizuka MN, Kolios MC, Sherar MD. A theoretical comparison of energy sources - microwave, ultrasound and laser - for interstitial thermal therapy. Phys Med Biol 1998;43:3535-47.

31. Strickland AD, Clegg PJ, Cronin NJ, et al. Experimental study of large-volume microwave ablation in the liver. Br J Surg 2002;89: 1003-7.

32. Strickland AD, Clegg PJ, Cronin NJ, Elabassy M, Lloyd DM. Rapid microwave ablation of large hepatocellular carcinoma in a high-risk patient. Asian J Surg 2005;28:151-3.

33. Meredith K, Lee F, Henry MB, Warner T, Mahvi D. Microwave ablation of hepatic tumors using dual-loop probes: results of a phase I clinical trial. J Gastrointest Surg 2005;9:1354-60.

34. Simon CJ, Dupuy DE, Iannitti DA, et al. Intraoperative triple antenna hepatic microwave ablation. Am J Roentgenol 2006;187:333-40.

35. Poon RT, Ng KK, Lam CM, et al. Learning curve for radiofrequency ablation of liver tumors: prospective analysis of initial 100 patients in a tertiary institution. Ann Surg 2004;239:441-9.

36. Iannitti DA, Martin RC, Simon CJ, et al. Hepatic tumor ablation with clustered microwave antennae: the US phase II trial. HPB 2007;9:120 4.

37. Topal B, Hompes D, Aerts R, Fieuws S, Thijs M, Penninckx F. Morbidity and mortality of laparoscopic vs. open radiofrequency ablation for hepatic malignancies. Eur J Surg Oncol 2007;33:603-7.

38. Sun Y, Wang Y, Ni X, et al. Comparison of ablation zone between 915 and $2,450-\mathrm{MHz}$ cooled-shaft microwave antenna: results in in vivo porcine livers. Am J Roentgenol 2009;192:511-4. 